\title{
Formation models for cosmic ray antinuclei
}

\section{Kachelrieß, ${ }^{a}$ S. Ostapchenko ${ }^{b}$ and J. Tjemsland ${ }^{a, *}$}

a Institutt for fysikk, NTNU, Trondheim, Norway

${ }^{b}$ D.V. Skobeltsyn Institute of Nuclear Physics, Moscow State University, Moscow, Russia

E-mail: jonas.tjemsland@ntnu.no

The expected low background of light antinuclei, such as antideuteron and antihelium-3, makes them ideal detection channels for exotic physics, such as dark matter annihilations. At the same time, their small binding energies and composite structures make them promising probes for the QCD phase diagram in heavy ion collisions. In order to correctly interpret experimental data, however, a solid description of the process of antinuclei formation process is needed. This can be achieved using phase space coalescence models based on the Wigner function representation of the produced nuclei states. Here, we discuss topics related to the production of light (anti)nuclei with a focus on its relevance for cosmic ray studies. In particular, we consider the importance of including both two-particle correlations and the size of the formation region on an event-byevent basis when describing the production in small interacting systems, such as $e^{+} e^{-}, p p, p \mathrm{~N}$ and peripheral NN collisions. As such, we review the newly developed WiFunC model (Wigner Functions with Correlations) and comment on its generalisation to larger interacting systems.

$37^{\text {th }}$ International Cosmic Ray Conference (ICRC 2021)

July 12th-23rd, 2021

Online - Berlin, Germany

\footnotetext{
${ }^{*}$ Presenter
} 


\section{Introduction}

Cosmic ray antinuclei have for a long time been considered a ideal probe for new and exotic phyics due to the suppressed background at small kinetic energies (see Ref. [1] for a recent review). In fact, a reliable detection of even a few low energy antinuclei can be considered as a proof of new physics, such as dark matter annihilations. Despite of intense searches, no detection has yet been confirmed. However, with the ever-growing statistics of AMS-02 and the upcoming GAPS experiment, a detection is conceivably around the corner. At the same time, the small binding energies and composite structures of light antinuclei make them promising probes for the QCD phase diagram in heavy ion collisions. In order to correctly interpret experimental data, however, a solid description of the formation process is needed.

This proceedings article is mostly based on Refs. [2-4]. There, we introduced an improved coalescence model based on the Wigner Function representation of the nucleons and the produced nucleus and applied it to astophysical processes and recent collider data. ${ }^{1}$ Here, we discuss formation models for cosmic ray antinuclei with a focus on the WiFunC model and its relevance for cosmic ray studies. For concreteness, the focus will be on antideuterons, but the same considerations apply to all kind of light nuclei with small binding energies, such at helium-3 and tritium.

\section{The coalescence model in momentum space}

In small interacting systems relevant for cosmic ray studies, such as dark matter annihilations or $p p$ collisions, the production of (anti)nuclei is often described using coalescence models in momentum space. Here, two (or more) nucleons coalesce into a nucleus if their relative momentum difference is smaller than the coalescence momentum, $p_{0} \cdot{ }^{2}$ As the coalescence model is purely phenomenological, $p_{0}$ has to be fitted to experimental data. Traditionally, the model has been expressed via the parametrisation of an invariant coalescence factor $B_{A}$ as

$$
\frac{\mathrm{d}^{3} N_{A}}{\mathrm{~d} P_{A}^{3}}=\left.B_{A}\left(E_{p} \frac{\mathrm{d}^{3} N_{p}}{\mathrm{~d} p_{p}^{3}}\right)^{Z}\left(E_{n} \frac{\mathrm{d}^{3} N_{n}}{\mathrm{~d} p_{n}^{3}}\right)^{N}\right|_{P_{A} / A=p_{n}=p_{p}},
$$

which relates the yield of a nucleus with mass number $A$ to the yield of its contitutents of $A$ protons and $N$ neutrons. The coalescence momentum is related to the coalescence factor as

$$
B_{A}=A\left(\frac{4 \pi}{3} \frac{p_{0}^{3}}{m_{N}}\right)^{A-1}
$$

in the limit of isotropic nuleon yields. However, since the yields in small interacting systems are highly non-isotropic, the coalescence model should be improved by taking into account two-particle momentum correlations by considering the coalescence condition on an event-by-event basis using a Monte Carlo event generator [5, 6].

\footnotetext{
${ }^{1}$ The discussions regarding the formation process are equally valid for particles and antiparticles, and the prescription 'anti' will thus often be dropped.

2 This assumption is motivated by the small binding energy of light nuclei. The nucleus is therefore formed through the process $N_{1} N_{2} \cdots \rightarrow A^{*}$ in this model, and the exitation energy is often assumed to be released later.
} 
The nucleus yields will in general depend on the full phase space density of the coalescing nucleons. When the coalescence condition is evaluated only in momentum space, it is no surprise that the coalescence momentum becomes process dependent. In particular, one will expect $p_{0}^{e^{+} e^{-}} \gtrsim$ $p_{0}^{p p} \gtrsim p_{0}^{p \mathrm{~N}}$ due to the increasing interaction region. This is behaviour is clearly seen in the left part of Fig. 1 where the values of $p_{0}$ obtained by fits to to various collider data and different event generators are plotted as a function of energy $[2,3,7]$. In fact, due to the apparent smooth behaviour of $p_{0}$ in this plot, it was suggested in Ref. [7] that $p_{0}$ exhibits an energy dependence. However, as we will see later, this apparent energy dependence can instead be explained purely by the expected process dependence.

In heavy ion collisions, the coalescence condition is typically evaluated in position space rather than in momentum space. In this case case the coalescence factor instead scales with the nucleon emission volume as $B_{A} \propto V^{A-1}$. There has been a lot of efforts made to unify these approaches using e.g. Wigner functions $[8,9]$. A key observation in these studies is that the emission volume probed in femtoscopy correlation measurements can be directly related to the coalescence probability [9]. This idea was recently explored for $p p$ collisions in cosmic ray [10] and LHC [11, 12] studies. In Ref. [12] it was argued that the success of the femtoscopy framework is stong evidence that coalescence is a main production mechanism for (anti)nuclei.

\section{The WiFunC model}

Motivated by the apparent process dependence of $p_{0}$ and the inacurate description of experimental data observed with the standard coalescence model, we deloped in Ref. [2] a new per-event coalescence model that takes into account both two-particle correlations and the nucleon emission volume in a semi-classical picture. This model was later refined and applied to cosmic ray studies and recent LHC data in Refs. [3, 4] (see also Ref. [13]). We will be using the abbreviation WiFunC (Wigner Functions with Correlations) for this model.

The main idea behind the WiFunC model is to use the Wigner Function approach already applied in the heavy ion community for decades (see e.g. Refs. [8, 9]), but to keep momentum correlations. In this way, both momentum correlations and the size of the formation region can be considered simultaneously. Momentum correlations should be taken into account due to the highly non-isotropic particle distributions and small multiplicities in small interacting processes. Meanwhile, the importance of including the emission volume even in point-like processes can be understood by considering the time-scales of the problem: The initial hard scattering is nearly pointlike since it occurs on time-scales $\sim 1 / \sqrt{s}$. The succeeding perturbative cascade proceeds with a characteristic momentum transfer $\Lambda_{\mathrm{QCD}}^{2} \ll\left|q^{2}\right| \leq s$. This means that the spread in the longitudinal direction (relative to the particle beam) is governed by the hadronisation length, $L_{\text {had }} \sim \gamma \sigma_{\|}$, with $\sigma_{\|} \sim 1 \mathrm{fm}$. On the other hand, the transverse spread will be related to the random walk of the perturbative cascade, $\sigma_{\perp} \sim \Lambda_{\mathrm{QCD}}^{-1} \sim 1 \mathrm{fm}$. These are close to the size of light nuclei of a few femtometers.

In the WiFunc model, one can write the probability that a given proton-neutron pair coalesce as

$$
w=3 \zeta_{1} \Delta \mathrm{e}^{-d_{1}^{2} q^{2}}+3(1-\Delta) \zeta_{2} \mathrm{e}^{-d_{2}^{2} q^{2}}
$$


where

$$
\zeta_{i}=\sqrt{\frac{d_{i}^{2}}{d_{i}^{2}+4 \tilde{\sigma}_{\perp}^{2}}} \sqrt{\frac{d_{i}^{2}}{d_{i}^{2}+4 \sigma_{\perp}^{2}}} \sqrt{\frac{d_{i}^{2}}{d_{i}^{2}+4 \sigma_{\|}^{2}}},
$$

and $\tilde{\sigma}_{\perp} / \sigma_{\perp}=m / m_{T}$ is introduced to account for the effective Lorentz transformation of the transverse spread important at large $p_{T}$. A similar expression exists for helium-3 and tritium [2]. The expression in Eq. (3) is obtained by projecting the two-nucleon density matrix onto the deuteron density matrix and using a Gaussian as the ansatz for the spatial spread of the nucleons, as explained in detail in Ref. [2]. The weight has two terms because a two-Gaussian wave function was used to approximate the deuteron wave function to get an analytical expression, which effectively has the interpretation as a long-range and short-range interaction. In this way, $\Delta=0.581, d_{1}=3.979 \mathrm{fm}$ and $d_{2}=1.810 \mathrm{fm}$ can be determined from independent measurements of the deuteron wave function. The nucleon momentum in the nucleus rest frame, $q$, and the transverse nucleus mass, $m_{T}$, have to be evaluated on an event-by-event basis using an event generator.

The free parameters of the model, $\sigma_{\perp}$ and $\sigma_{\|}$, have the physical interpretation as the size of the emission region of nucleons in respectively the transverse and longitudinal direction relative to the particle beam. As discussed above, these are expected to have similar values in point-like processes and we therefore set $\sigma \equiv \sigma_{\perp}=\sigma_{\|}$to reduce the number of free parameters. Furthermore, the spreads will in general have a point-like contribution and a geometrical contribution from multiple parton-parton interaction. In the particular case of $e^{+} e^{-}$and $p p$ collision, we assume $\sigma_{\left(e^{ \pm}\right)} \simeq \sigma_{(p p)} / \sqrt{2}$ since the geometrical in $p p$ collisions are of the same size as the spread in pointlike processes. ${ }^{3}$ It is important to emphasise that although the model has free parameters, they can be well constrained. In fact, from the physical interpretation, we expect $\sigma_{\left(e^{ \pm}\right)} \simeq \sigma_{(p p)} / \sqrt{2} \simeq 1 \mathrm{fm}$. This is exactly what is observerd when fitting the model to experimental data, as seen in Fig. 1. In this plot, the process dependence is naturally taken into account, and the process dependence observed in the coalescence model in momentum space is completely gone.

Thus far we have focused on antinucleus production in small interacting systems. However, the WiFunC model can be applied regardless of the underlying process and physics as long as the event generator describes well the hadron production. For processes involving nuclei, one must in any case take more care in determining the spreads $\sigma_{\|}$and $\sigma_{\perp}$ since the geometrical contribution will vary event-by-event. For $p p$ collisions, this variation is weak and may be neglected. On the other hand, for $p \mathrm{~N}$ and $\mathrm{NN}$ collisions, the geometrical contribution will depend strongly on the rate of multiple scatterings in the event, and thus on the centrality, or the multiplicity, of the collision. For example, peripheral interactions at large impact parameters are dominated by the collision of nucleon pairs, implying that $\sigma_{\|}^{\text {(geom) }} \sim R_{p}$ like in the $p p$ case.

As a final note, also space-time correlations can in principle be taken into account in the WiFunC model by utilising the semi-classical space-time picture implemented in event generators like Pythia [14] or UrQMD [15]. In such cases, one should use the coalescence probability $w=3 \Delta \mathrm{e}^{-r^{2} / d_{1}^{2}-d_{1}^{2} q^{2}}+3(1-\Delta) \mathrm{e}^{-r^{2} / d_{2}^{2}-d_{2}^{2} q^{2}}$, where $r$ is the distance between the nucleons in the pair rest frame. In essence, this approach swaps the Gaussian ansatz' made on the nucleon distributions with the semi-classical treatment in the event generators.

\footnotetext{
${ }^{3}$ When more accurate event generators and experimental data become available, a seperate fit of the spreads may be neccessary.
} 

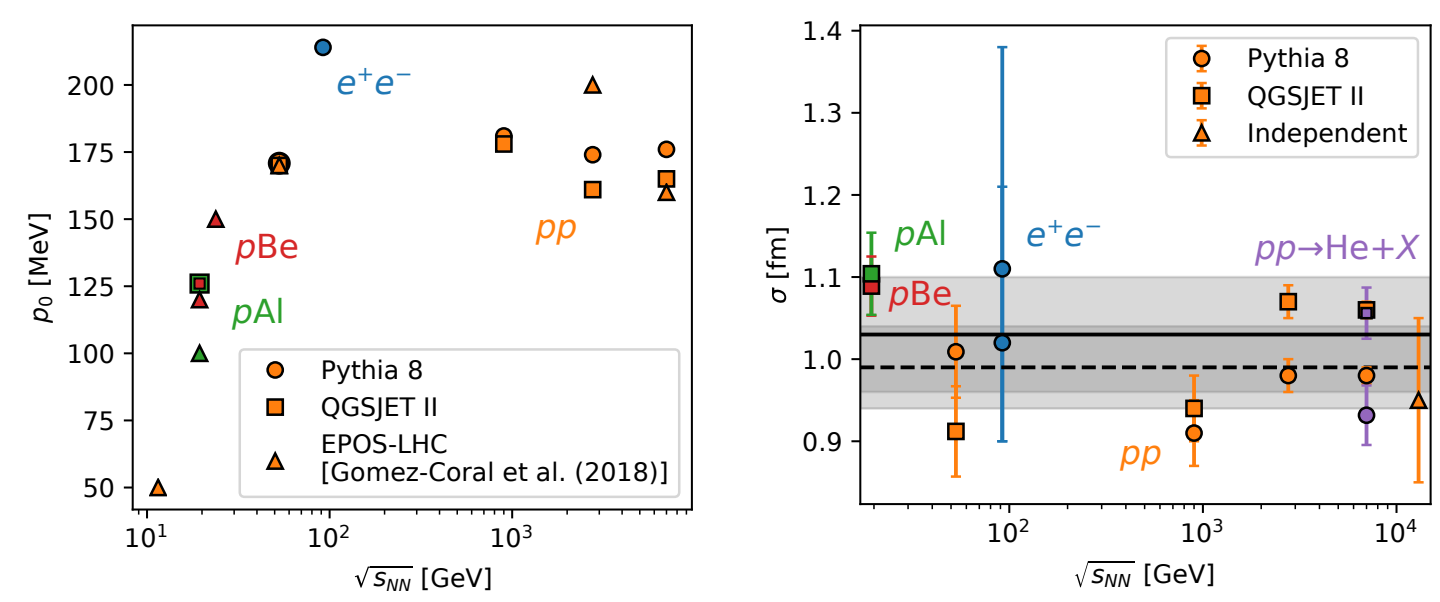

Figure 1: Coalescence momentum $p_{0}$ in the per-event coalescence model in momentum space (left) and parameter $\sigma$ in the WiFunC model (right) obtained by fit to experimental data using different event event generators. The data are taken from Refs. [3, 4, 7]. The figures are adapted from Ref. [13].

\section{Alternative models}

Although the coalescence model is the most widely used (anti)nucleus production model in cosmic ray studies, alternative models exist. A competing approach often used in for heavy ion collisions is based on statistical thermal models [16]. In these models, it is assumed that hadronisation and the formation of light nuclei is occuring at chemical freezeout in an expanding "fireball" of quark gluon plasma. Such models are motivated by the fact that light nuclei spectra in such collisions are consitent with a thermal production with a similar freeze-out temperature as mesons and nucleons. One major drawback of these models is that it is severely difficult to explain how the nuclei can survive the freeze-out process given their low binding energies. Furthermore, one should note that the coalescence picture implies that the energy spectrum of the nucleus is up to a quantum mechanical correction factor inherited by the nucleus [17], and the observed quasi-thermal spectra are therefore explained well by the coalescence picture.

Interestingely, recent collider data on small interacting systems, such as $p p$ and $p \mathrm{~Pb}$ collisions, have features characteristic for collective flows and the formation of a quark gluon plasma [18]. This has motivated the suggestion that the production of light nuclei even in small interacting systems can be described using thermal models [1,19-21]. One should thus mention that many of these-including the behaviour of the coalescence factor $B_{2}$ as a function of multiplicity [21] and transverse momentum in $p p$ collisions and the decrease in the baryon emission volume with transverse momentum [22] — are naturally described by the WiFunC model [4]. In any case, current evidences for the production of a quark gluon plasma and collective motion in $p p$ collisions are irrelevant for cosmic ray antinuclei studies since they are only observed at LHC energies.

Another approach worth mentioning is that of Ref. [23] (see also Ref. [24]). There, the production is modelled as a dynamical process in which the formation occurs in a final state interaction between nucleons, $N N \rightarrow X d$, where $X$ may be e.g. a pion on a photon. The coalescence probability is in turn assumed to be proportional to the corresponding scattering cross 
sections. This approach has the advatage that the excess energy and momentum in the standard coalescence models are naturally taken into account, but it is a phenomenological approach that does not take into account the nucleon emission volume and has to be fitted to experimental data.

\section{Relevance for cosmic ray studies}

In cosmic ray studies, we are mainly interested in the bulk of produced (anti)nuclei in particle collisions. For example, antinuclei are produced in so-called secondary interactions in which (mainly) primary protons collide with the interstellar matter consisting of (mainly) protons. The bulk of antideuterons are produced in interactions with primary energies $E_{\text {prim }} \sim 20-100 \mathrm{GeV}$ and have relatively large longitudinal momentum. In contrast, experimental data at LHC are only available at central (pseudo-)rapidity and thus large $p_{T}$. Therefore, it is important to utilise a production model with predictive power when studying cosmic ray antinuclei, such as the WiFunC model.

The isotropic coalescence model (1) with a constant $B_{A}\left(p_{z}\right)$ is still regularily applied in the literature in describing the formation of antinuclei in secondary production. This is motivated by the observation that a constant $B_{A}\left(p_{z}\right)$ is a good approximation for high energy collisions at LHC. In particular, in Ref. [10] femtoscopy correlation experiments are used to deduce a constant value for $B_{A}$, thereby avoiding the need for additional theoretical inputs. In Fig. 2 the value of $B_{2}\left(p_{z}\right)$ in $p p$ collisions obtained using the WiFunC model is compared to the one inferred from femtoscopy experiments [10] for energies relevant for cosmic ray and collider studies. As is readily seen, the approximation of a constant $B_{2}$ is valid at collider energies, but the data from LHC overestimate the production at cosmic ray energies. More importantly, the coalescence factor will depend strongly on the longitudinal momentum at the relevant energies, and a careful treatment taking into account two-particle correlations is thus required to obtain correct energy spectra.

\section{Conclusion}

Light antinuclei, like antideuteron and antihelium-3, are ideal detection channels for exotic physics in cosmic ray studies and the study of the QCD phase diagram in heavy ion collisions. In order to correctly interpret the experimental results, a solid understanding and description of the production mechanisms is needed. Therefore, we developed a per-event coalescence model that takes into account both two-particle momentum correlations and the nucleon emission volume in a semi-classical approach. This model improves several limitations found in existing coalescence models, such as apparent process and energy dependencies, and inacurate description of data.

\section{References}

[1] P. von Doetinchem et al., Cosmic-ray Antinuclei as Messengers of New Physics: Status and Outlook for the New Decade, JCAP 08 (2020) 035 [2002. 04163].

[2] M. Kachelrieß, S. Ostapchenko and J. Tjemsland, Alternative coalescence model for deuteron, tritium, helium-3 and their antinuclei, Eur. Phys. J. A56 (2020) 4 [1905. 01192]. 


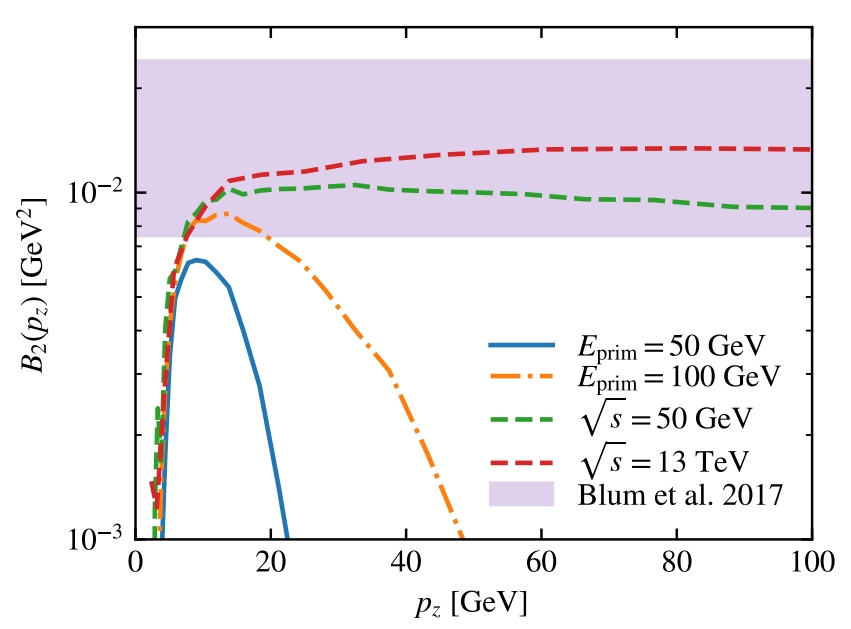

Figure 2: The deuteron coalescence factor $B_{2}$ as a function of longitudinal momentum in $p p$ collisions for various energies relevant for cosmic ray processes and collider studies is computed with the WiFunC model. The results are compared to the value in [10] deduced from femtoscopy experiments at LHC energies. Note that $p_{z}$ is given in the rest frame of the target. The figure is taken from Ref. [4].

[3] M. Kachelrieß, S. Ostapchenko and J. Tjemsland, Revisiting cosmic ray antinuclei fluxes with a new coalescence model, JCAP 08 (2020) 048 [2002 . 10481].

[4] M. Kachelriess, S. Ostapchenko and J. Tjemsland, On nuclear coalescence in small interacting systems, Eur. Phys. J. A 57 (2021) 167 [2012 . 04352].

[5] M. Kadastik, M. Raidal and A. Strumia, Enhanced anti-deuteron dark matter signal and the implications of PAMELA, Phys. Lett. B683 (2010) 248 [0908 . 1578].

[6] L. A. Dal, Antideuterons as signature for dark matter, master's thesis, NTNU Trondheim, available at http://hdl . handle. net/11250/246403, 2011.

[7] D.-M. Gomez-Coral, A. Menchaca Rocha, V. Grabski, A. Datta, P. von Doetinchem and A. Shukla, Deuteron and Antideuteron Production Simulation in Cosmic-Ray Interactions, Phys. Rev. D98 (2018) 023012 [1806. 09303].

[8] H. Sato and K. Yazaki, On the coalescence model for high-energy nuclear reactions, Phys. Lett. B 98 (1981) 153.

[9] R. Scheibl and U. W. Heinz, Coalescence and flow in ultrarelativistic heavy ion collisions, Phys. Rev. C59 (1999) 1585 [nucl-th/9809092].

[10] K. Blum, K. C. Y. Ng, R. Sato and M. Takimoto, Cosmic rays, antihelium, and an old navy spotlight, Phys. Rev. D96 (2017) 103021 [1704.05431].

[11] K. Blum and M. Takimoto, Nuclear coalescence from correlation functions, Phys. Rev. C 99 (2019) 044913 [1901.07088]. 
[12] F. Bellini, K. Blum, A. P. Kalweit and M. Puccio, On coalescence as the origin of nuclei in hadronic collisions, 2007.01750.

[13] J. Tjemsland, Formation of light (anti)nuclei, PoS TOOLS2020 (2021) 006 [2012 . 12252].

[14] S. Ferreres-Solé and T. Sjöstrand, The space-time structure of hadronization in the Lund model, Eur. Phys. J. C 78 (2018) 983 [1808.04619].

[15] S. Sombun, K. Tomuang, A. Limphirat, P. Hillmann, C. Herold, J. Steinheimer et al., Deuteron production from phase-space coalescence in the UrQMD approach, Phys. Rev. C 99 (2019) 014901 [1805.11509].

[16] A. Andronic, P. Braun-Munzinger, K. Redlich and J. Stachel, Decoding the phase structure of QCD via particle production at high energy, Nature 561 (2018) 321 [1710.09425].

[17] L. Csernai and J. I. Kapusta, Entropy and Cluster Production in Nuclear Collisions, Phys. Rept. 131 (1986) 223.

[18] J. L. Nagle and W. A. Zajc, Small System Collectivity in Relativistic Hadronic and Nuclear Collisions, Ann. Rev. Nucl. Part. Sci. 68 (2018) 211 [1801.03477].

[19] F. Bellini and A. P. Kalweit, Testing production scenarios for (anti-)(hyper-)nuclei and exotica at energies available at the CERN Large Hadron Collider, Phys. Rev. C 99 (2019) 054905 [1807.05894].

[20] J. Cleymans, S. Kabana, I. Kraus, H. Oeschler, K. Redlich and N. Sharma, Antimatter production in proton-proton and heavy-ion collisions at ultrarelativistic energies, Phys. Rev. C 84 (2011) 054916 [1105.3719].

[21] ALICE collaboration, (Anti-)deuteron production in pp collisions at $\sqrt{s}=13 \mathrm{TeV}$, Eur. Phys. J. C 80 (2020) 889 [2003.03184].

[22] ALICE collaboration, Search for a common baryon source in high-multiplicity pp collisions at the LHC, Phys. Lett. B 811 (2020) 135849 [2004.08018].

[23] L. A. Dal and A. R. Raklev, Alternative formation model for antideuterons from dark matter, Phys. Rev. D91 (2015) 123536 [1504.07242].

[24] P. C. Gugelot and S. M. Paul, Deuterons from 300-GeV proton collisions calculated from the Lund model, Z. Phys. A 344 (1993) 325. 